\title{
Le contrôle-commande des petites centrales
}

\author{
Small hydropower stations control and monitoring \\ par P. Jacquemmoz, D. Tironi \\ Electricité de France
}

\begin{abstract}
An hydropower station control and monitoring system is in charge of electromechanical devices automatic working. Unil now, the EDF small stations control and monitoring was based on the other stations systen. But today, in order to reduce the global cost of a station, the principles for the small stations that do not jeopardize people or electrical network, are to set up only working specifications and a guide for electrical installation. These new principles have been applied to some small stations renewing in 1997, in particular the Rabastens station.
\end{abstract}

\section{I 口 AMÉNAGEMENT HYDROÉLECTRIQUE}

Un aménagement hydroélectrique transforme l'éncrgic naturelie hydraulique en éncrgic électrique produite dans les alternaleur's.

Il est constitué d'une succession d'ouvragges qui participent directement à la production élecurique : les ouvrages de retenue (les barrages, priscs d'eau), d'amenée d'eau (les galerics, canaux, conduites forcées), de production (turbines alternateurs), d'évacuation d'énergice (transformateur, disjoncteurs).

\section{EONTRÔLE-COMMANDE}

1] est indispensable de coordonner le fonctionnement des équipements électromécaniques. Ainsi le démarrage d'un groupe de production (ensemble urbine alternateur) nécessite l'enchaînement parfaitement maîtrisé d'opérations élémentaires : ouverture de la vanne de garde, ouverture du vannage, misc en rotation progressive, excitation de l'alternateur; " couplage " avec le réseau électrique général.

Les centrales ćtant automatiques, c'est le contrôle-commande de groupe qui gère toutes ces actions.

Un système automatisé est composé dc dcux parties :

- la partic commande, autrement dit le contrôje-commande ; - la partie opérative, autrement dit le processus physique à commander (partie operative d'un groupe de production, des services généraux etc.).

La partie opérative d'un groupe comprend la vanne de garde, la turbine, l'alternateur, éventucllement le transformateur, le disjoncteur, les origanes auxiliaires du groupe.

Les capteurs (contacts lins de course, captcurs de mesure) ct les actionneurs (électrovannes, contacteurs) sont usuellement inclus dans la partic opérative.

La partie commande comprend l'automatisme de démarrage/arrêt, l'asservissement aux consignes et la mise en sécurité du groupe, la commande des organes, les signalisations et alarmes ćventuclles.
La frontière entre les purrties opérative et commande d'un groupe est généralement situće au niveau des câbles de liajson entre ces deux parties.

\section{III — HISTORIQUE}

Dans les annécs 70, EDF a édicté un corps de doctrines techniques pour le contrôle-commande des groupes de production. Ces doctrines accompagnaient la généralisation des centrales automatiques sans personnel de conduite. Elles reposaient sur les principes directcuss suivants, toujours en vigueur:

- Ie fonctionnement normal est automatique ;

- la tension de contrôle-commande est fournic par une batterie unique $125 \mathrm{~V}$ ct distribućc selon une tranche continue par groupe ;

- l'état de sécurité d'un groupe est l'arêt ;

- l'état de sécurité d'une centrale dépend des conditions hydrauliques ;

- less schémas sont à manque de tension.

Les automatismes des groupes ont été réalisés dans le respect des ces principes à partir de schémas types à relais. D'autre part EDF a engagé des programmes de tests en vue de qualificr les matériels critiques pour la sécurité des installations : relais de protection, automates de couplage, chaînes de mesure de vitesse, diffuseurs d'alarmes, automates de consignation des événements...

Dans les années 80 les automates programmables industriels se sont progressivement imposés lors des opérations de rénovation. Des "Grafcets" types ont remplacé les schémas à relais dans le corps de doctrine, une architecture de contrôlê-commande a été définie pour les groupes de puissance unitaire inférieure à $30 \mathrm{MW}$. Des gammes d'automates programmables ont été qualifiées.

Depuis 90, l'emploi des automates programmables a été confirmé pour les groupes de puissance supéricure à $30 \mathrm{MW}$.

Ces doctrines ont permis de bâtir des automatismes 
simples, fiables, disponibles. facticment maintemáles par les atgents di'exploisation.

Cependant les antomatismes des jetites centralies décou laient des principes appliqués sur les antres installations avec quelques simplifications, notamment dans le plan de protection des machines.

\section{ILS CENTRALES $\Lambda$ ANJEL PLREMENT ÉCONOMIQLE}

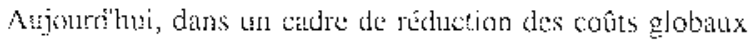
(investissement et axplotiation sur la durée be vie des instal lationsi de nouvelles orsertations lichnichues allégécs visent

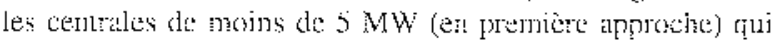
n'on pas détinpact sur la sécurtú publique (présence d'un

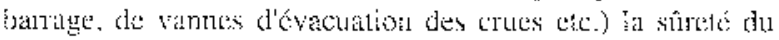

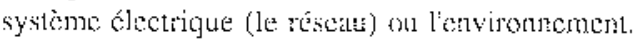

Ces centrales, clites à enjeu purement économique, fonctommert ilutomatiquement al fil de l'étu. Lil melinternance en cst prioriatement corrective

I.es prescriptions EDF relatives an contrôlc-iomenande se

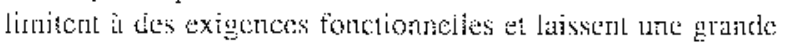
latitude atix entreprises réulisatrices pout proposer des solutions tectmiquess cononiques.

Les equipements doivent fester nodulares el simples. I.t remplatement dun équipernent élémentairs ou d'un constitit:st fiendiate lit dure de vie de l'installation de contrôle commande chot se faje par sthstitution sans impace sur !cs autres equipe Iyents, ce qui garantil lintcroharyeabilité des composants.

Les logiciels son structures et modufaires. On privileger l'atilisation d'outils pararnétrables par rappon ì des dévelop penents spécifianes

\subsection{Pilotage de la centrale}

\subsubsection{Conduite}

Sont assurés:

la conduite antonatique de la centrane an limitant les gradients des dépits sortant lors des changements de régime; ; n particulicr, la rëtrulation dia nivean toit rechercher un comn promise entre les bearts de mivealu et les variations de débit sortant ;

- l'enchatnement des dérnarrages al arêts des groupes en teriesst compte des indisponjitilités el de lá consiṣne de débit à répatir tout en minimisantl le nombre de sthlicitations sur les machines.

\subsubsection{Protetctions}

Sont alsourés :

- Le tratienerat des profections des services gérérallx et des átuxiliaires (destribution d'auxiliaires, ateliers d'Énergie. ponipes ['exhature, vatnes de têtc...) :

- l'ank̂t d'urgence général ;

- le respect du plaln de protection imposé par l'cxploitant du réseau électrigue on or qui conceme les délants extóricurs à la centraic $(\mathrm{OIE} 2606 \ldots)$.

\subsection{3 hiterface " hommes mathine."}

I.'interface utilisatcur conpret. seulement las commandes cosentielles (arrête duręzence, sćfection de mode de marche et ordres de commande des grompes, sélection de mode de Inarhe et ordres de commande doryanes aluxiliaires) it les signalisations indisperisab)es (dletant groupe, mesure de ptis sance, nivealn de la retenue).

\subsubsection{Simneillance?}

I a contrôte-commande gère les alatmes st leur ómission au persominel (alerte locale si présence de personmel à lá centrale, renvoi cl'alarmes à distance, possibilits d'acquattement

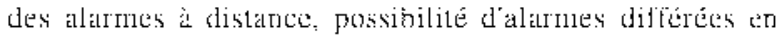
heures ouvables...), la cosisignation des évémements (défeuts, alarmes et actions d'exploitation el restitulion des messages bocalenent voirc à distance), l'exploitation ì di.s tance tyâce à un dispositif simple permeteart de consutter ì distastce certaimes informations ê đ'intcrvenir su: fe déroule ment du processus on marche normali.

rouy' les organcs cssemticls comme disjoncteur de groupe et [igne, vannages. vannes de tête, varnes de gatude, le resour

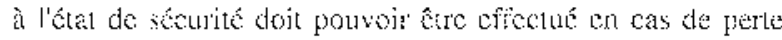
totale d'une sourese d'alimentation électrique auxiliaire.

\section{: 4.2 Pilotage des groupts}

I.es groupes ne diknosent mi de rétulation de vitesse, ni des rógulatjon de terision; en vue de resjecter les exigentes sur

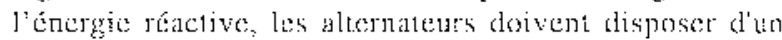
réglage de l'excitation et les gérératrices asynchrores d'unt: compensation de factent de puissance.

\section{Sont assurćs :}

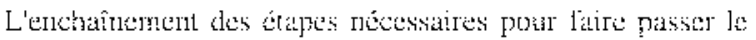
groupe de l'artêt à la marche étáblic cn turbine et vice-versal darts le cas d'arrêt normal ot sur défaut. Un groupe fonctionne on marche automatique enchaîne ou $\mathrm{cm}$ marche sem atstio malicae. Dans as dernier eas, lexploitant agit manuellement

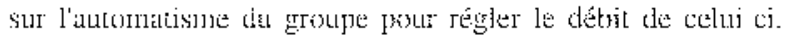
Lne possibilité de démarrage fractionné est demandéc.

- La protection sir défauts électriques : découplage et desexcitation immédiatis, sitvis d'une fermetute mpide de l'ortgane d'adrsission du débir el de l'arrêt blogué du groups.

Is protection sur diffalts mécanciges : Cermeture rapide de l'organe d'âdrnission du débit pais découplage, désexcitá tim et atrêt bloguté dị groupe.

- T a protection sur défauts réseau : découplage o désexcita lion immédials, suivis d'une Cermelure rapide de l'organe

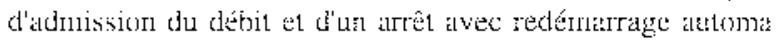
tigue des que les conditions de réseau et hydrauliques le jee melterit.

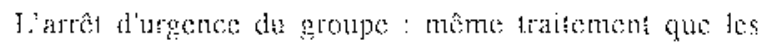
protections électriques.

I a conmande et l'asservissement des organes réplants aux valeurs de consigne.

Les protections som imposéces par l'explosiant IDDI.

\subsection{Les services auxiliaires}

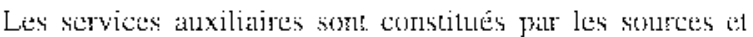
les circuits de distrilution d'énergic à courant altenatil et it courant. continu néeessaires nour assurer le fonctionmement des instaliations de l'ouvinge.

11 ext exigé unc seule source il courant allerratit, avec

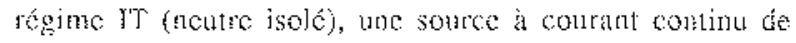
$24 \mathrm{~V}$ orl $48 \mathrm{~V}$.

\section{$V$ — EXPÉRIMENTATION : RÉNOVATION DE IA CENTRAI.E DE RABASTENS (1997)}

I al mâ̂trise diouve est réalisée par le Service Ingénicric Hydraulige d'E.D.F. USI Suc-Ouest. 


\begin{tabular}{|c|c|c|c|}
\hline \multicolumn{2}{|r|}{ Repère groupe } & G1 & G2 \\
\hline \multicolumn{2}{|r|}{ Année mise en service } & 1928 & 1950 \\
\hline Turbine & $\begin{array}{r}\text { Type } \\
\text { Débit } \\
\text { Petite vitesse } \\
\text { Renvoi óangle mutiplicateur vitesse } \\
\text { Grande vitesse } \\
\text { Puissance }\end{array}$ & $\begin{array}{l}\text { Hélice verticál } \\
15.3 \mathrm{~m}^{3} / \mathrm{s} \\
75 \mathrm{tr} / \mathrm{mr} \\
\text { engrenages à chevrorls } \\
428 \mathrm{tr} \mathrm{mr} \\
283 \mathrm{~kW}\end{array}$ & $\begin{array}{c}\text { Kaplan verical } \\
6,3 \mathrm{~m}^{3} / \mathrm{s} \\
185 \mathrm{tr} / \mathrm{mn} \\
\text { engrenages } \\
128 \mathrm{tr} . \mathrm{mn} \\
132 \mathrm{~kW}\end{array}$ \\
\hline Altermateur & $\begin{array}{r}\text { Type } \\
\text { Puissance plaque à borne } \\
\text { Puissance maxi fournie sois } 3 \mathrm{~m} 60 \text { de chute } \\
\text { Tension nominale } \\
\text { Vitesse } \\
\text { Excitation }\end{array}$ & $\begin{array}{l}\text { Axe horizonlal } \\
225 \mathrm{kVA} \\
220 \mathrm{~V} \\
428 \mathrm{tr} / \mathrm{min} \\
\text { Stáiquic }\end{array}$ & $\begin{array}{l}\text { Axe horizontal } \\
225 \mathrm{kVA} \\
160 \mathrm{~kW} \\
220 \mathrm{~V} \\
428 \mathrm{tr} / \mathrm{mn} \\
\text { Statique }\end{array}$ \\
\hline
\end{tabular}

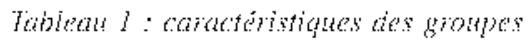

\subsection{Présentation succincte de l'amérraģeme'nt}

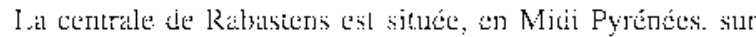
le cours infrien dir Tarn ontro Allij ct Toulonse. Elte est alimentse par un barrage de type digue dićversante. Ia banlen de chute maximale es! ce $3,6 \mathrm{~m}$. Le débil maximatal tur

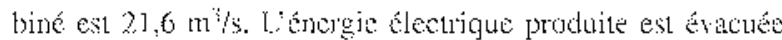
par une ligne E.G.S. $20 \mathrm{kV}$ (cf. Tablean l).

\section{\$.2 Présentation du projet.}

\subsubsection{Anpertise de leaistant}

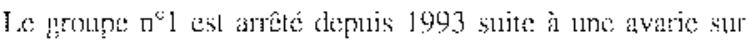
le multiplicatcur à engrenages four lequel les pièces de rebange ne somt plus babrinnces (plusieurs dicnts sont cassécs).

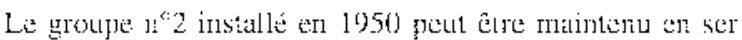
vice moyentant :

ure révision de fat roue,

- le remplanmen par un vétin cí régulateur de viesse

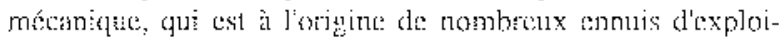
Lution.

\subsubsection{Solation retentut}

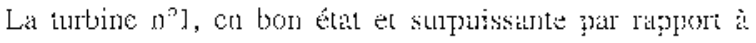
l'altenateut alu'el]e entraînait, se verra acouplé à uno génératrice asymchrone patr l'intemédiaire d'un nosvesu multipij catcur de vitesse.

Les circuits d'évacuation d'énergie, cellules $20 \mathrm{kV}$ instal kes en 1983 et transformatcus datan de 1967 scrone comistués.

Le contrôle-commande installé cr 1983 sur plate-forme strélevée est égulement à censerver moyennant une réfection de t'amoire de commande $G 1$ pour le nouverti groupe, des adaptations pour ic ( 32 at la renrise des services gínchand du fait de l'installation d'un antomate proglimmlitule.

Cote opération est envisanceable dans la mesure oi un gain est prévu sur le productible anmedel bu tait de l'agyment. tation de puissance du G1 aves un retom sur investissenent de 12 allis, estimlé par EDF Energie Midi Pyrérées.

Dans la suite de l'exposié, ne sera developpé que l'opération de rérnovation du eontrôle commande de lat centrale de Rabastens dans lo eadre des usines à enjeu purmen fonorniculus.
5.3 Rénovation des équîpements de contrôlenconnunde

5.3.1. Introduction

Le nusître not de cette operation ast : " réduction des conts". Dans ceth optique, ure certibu nombre de principes mกt été mišs en cruvre:

it Le nouveall contextc do la realisation,

bi Reciserelac des fondions minimales de comtôle-com mande el simpilicution des schtémas,

ci Stralígic diachat.

\subsubsection{Contexte de ia rediscition/exemples}

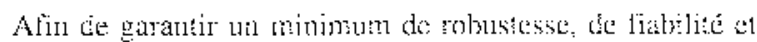
de mantenanes ies épupements atu-delà de la curéc de

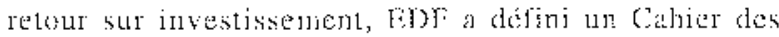

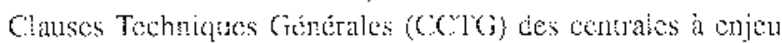

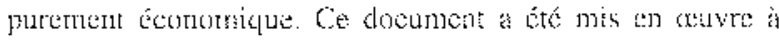
titre expérimental sur l'affaice a Rabastens.

LUH avee ce CCTG ne s'atliche plus en tarn que spécili calleur de materjel at laisse aux critroprises lo choix bas matericls. Nous alons montrer à j'aide de quelcyues exempyles comment le choix des matériels peut amener une tódhetion des: coût:.

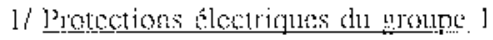

1a choix s'est porté sur an appareillage numsérique pro-

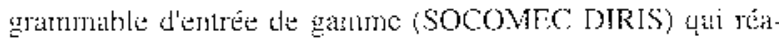
lise les protections blectrighes du grolipe. Cet apparcillage comporte anssi un éeren LCD de visualisation, permetant d'afticher les 3 courants, ]es 3 tensions, la pujswatace active el réactive: amsin que le curntu de production.

Pur lá lusion de 2 fonctions hasituellement séparécs (prolections / interface hommomachine) le gain en lorne de diminution de la fiferie et de l'HH: est injortant.

2/ Contrôle de teiugératar

Lu apparcillage numériṇue programmalyle a été choisi

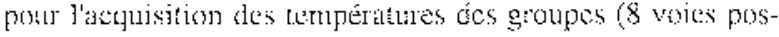
sibles). Le traitemcat des protections est progranmentable el róalisé par la fermeture d'une houcle sectice sut senils. I.c suivi à distance est possible grâne à l'existence dure lialisorn séric et la vistalisalion des températures sar chaque voic se läit par an écran digitall. 


\section{3/ Acquisition de la vitexse des grompes}

Cette fonction est nókessaine pout le contrôle du scéguentiel de dénthinge des groupes al fal protection de survitesse.

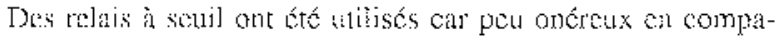

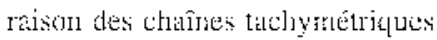

4 Copteur de mesure de la hanteur dean dans la reternes

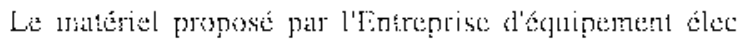

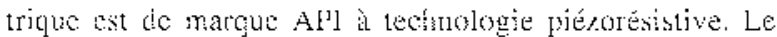

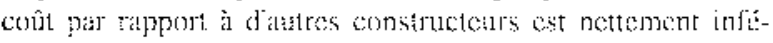
ricul (de l'orde de 2 à 3 ).

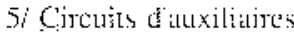

Partout où lá norre C.15.100 le permet, des lusibles ast

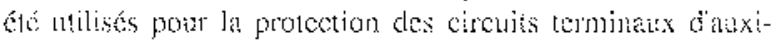
liaires à la place de disjonetents divisiontaires.

\section{$6 /$ Conctusion}

Cos quelcaues exemples indiquent bien que lo choix d'un

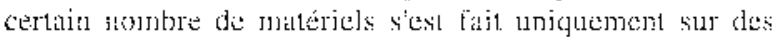
consideratons bomoniques. On peut s'intenoger sur !a fia-

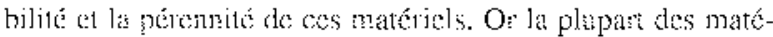
riels utilisés sont commus par now servicen édéjà tutilisés sur dos applications sams énjeu) et sost latrgement mis en (xurre par ties pooducleurs indépenciants.

Pour prolonger la logique dans laquelle est insorite do

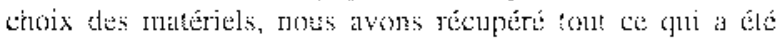

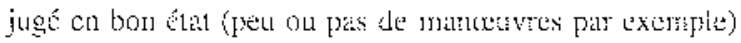

- banctles de commexion DAV,

- interrupteurs de tranctie.

courl circuiteurs,

boîtes d'essai type SICTIRA

disjoncteurs division:atres,

porte fusibles.

Ces1 un changenent de logique important darts nos ćlablissements biern que déjil réalisé par nos anciens.

\subsubsection{Simplification des fontion.}

De membreuses simplifications sur les fonctions die

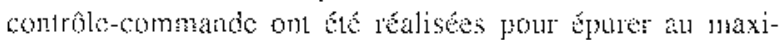
man tout ce qui nis ser pas strictement a la prodaction denergic.

I/ Interlace homme machinl

Celle-ci à été rédujte alu minimum. Pour de (j) nouk avoms mentionné comment nous y sommes parvenus (voir s. 5.3.2),

la fonction de consignation d'états ya pas été mise en place car elie nécessite une imprimante ot dono line mantenance. Fille a cé rériplacée par un produt irteme EDF yui permet de télé exploiter les anónagements à distance.

\section{2i Fonctions des services góneranx}

les circaits de détection d'incendic ont ćte supplines. Il y a cu simpliffrction de circuit des alarmes de façor ì rlavoir plusi culun seul type d'alarme transmis à la lête do groupenent dusines en heures ouvrables. Cest le logicied de télé exploitution qui permed de oćrer la transulission des alatmes.

\section{$3 /$ Architecture antomate}

I.'archilecture retenuc csi à un scul automate. Celui-ci rènplipl les fonctions suivanties:

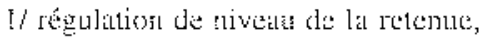

2/ répartition de ciarge sur las 2 groupes

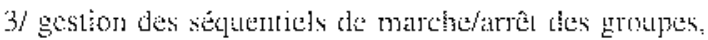

4) rolage en malrcice des machines:

5i aceuisitios: et traitement des protections des groupes.

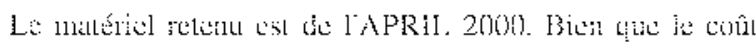

de ce matériel reste élevé uar rapport à d'autres alutomiates cetté solution a ćté choisise en règurd de :

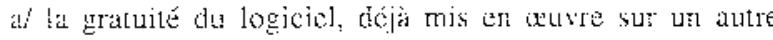
amćnagement similairé.

b/ la mantenance du jongejel, ear il s'agit d'an standarel en interse,

c/ la maintenance du mutériel, ca: lo groupment d'usines prosede du matériel ale rechange.

4 Schema de comtrobe counmandr.

pour les gounes, la schérnil de contôlo-commande retenu est à logique positive (schéma à śmission). Les protections électriques a mócaniques sont acequises par l'sutomate qui pilote par ses sorties les divers organes des groupes : dis joncteur; vantage/palcs, vanne de gurde...

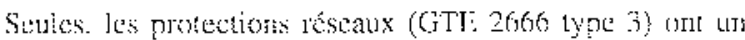
contéct doublé permettant le décientchement dos disjoncteurs des groupes

Les risques d'un tel schéma sont contrus ace aceptés an legard de l'enjou de l'usine (pus de rebontance, pas te retour à l'état léce sécurité par coupure d'une polarité...)

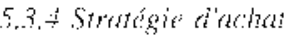

La réduction des conts ne peut jas s'appliguer uniquement

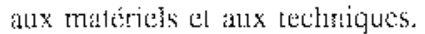

Lil travail de recherehes dientreprises capables de mencr à

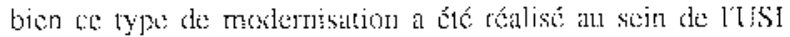
Sud Oesesc, Service Irgénierio Iydratulique et Service Achats.

I.a visite de nombreuses usines de producteurs hydrau liçues autonomes on industriels de lat région Midi-Pyrétoécs a

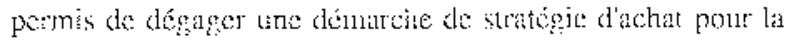
modemisuatien du contrôle-commansde d'usine ì enjet puretuent Économique. Fule a permis de répondre aux contraintes aconomigues des exp̧oitants pour lescuels 3 porsibilités se présententl :

ne pas senouveler l'abitorisation

arrêter lá cantrale el attendo la fin de l'aulorisation ou vendre.

rentouveler si les coûts de renouvellemerst sort retrtables.

I.es critères de sélection das entreprises ont été less suivallts:

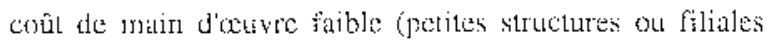
de grands groupes):

expérience dans le domaine avec des léférences,

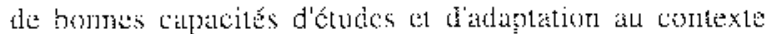
varis dos amentagements hydrauliques.

Le choix s'est porté sutr une PME ayant une grande expérience aupres de producteus autonomes die la vallé de l'sriège.

\section{CONCLESION}

Aures plusieurs décennies où le contrôlo-commando des

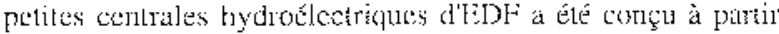
des spécifications applicables aux gratades centrales, les orientalions actuelles s'bcartant des doctrines techriques internes à l'entieprise et permetient des solutions technicuses orignales. dans l'objeetif d'une roduclion des coûts.

Ces orientations ont été expérimentées avec suecès à Rubaterts où l'instaliation intègre des fornctionnaklités simplifiécs. des matéricls écontomiques at une stratépie d'actalt (o) 